\title{
LOS CAMINOS DE LAS REFORMAS. LA VIRTUALIZACIÓN UNIVERSITARIA EN AMÉRICA LATINA
}

\author{
Claudio Rama ${ }^{1}$ \\ Universidad de la Empresa - Uruguay \\ claudiorama@gmail.com
}

Recepción: 07/10/2012

Evaluación: 29/19/2012

Aceptación: 15/11/2012

Documento - Revisión del Tema

doi: http://dx.doi.org/10.9757/Rhela.19.02

\section{RESUMEN}

En el presente documento se analiza el creciente proceso de incorporación de tecnologías de comunicación e información en la educación y de cómo está impactando los modelos previamente existentes semipresenciales. Se analiza como derivado de su impacto, las dinámicas universitarias están insertan en un fuerte proceso de virtualización. Se formula la hipótesis de una tipología los caminos, destacándose la reingeniería de las instituciones semipresenciales de educación a distancia; las nuevas ofertas de educación universitaria 100\% virtuales; la virtualización de segmentos puntuales de

\section{CHANGING PATHS" LATIN AMERICAN UNIVERSITIES GOING VIRTUAL}

\section{ABSTRACT}

Present paper analyses the growing process of using and incorporating information and communication technologies in education and how does this process impact in existing models of part time education processes. Resarcher analyses impact of processes, university dynamics and how these are affected by a constant and strong model on going virtual. Hypotesis is presented as la educación presencial y la informatización de la gestión administrativa. A partir de un análisis comparativo, se formula la hipótesis de la conformación de una lenta educación digital y la desaparición de las tradicionales distancias entre una educación a distancia y una educación presencial.

Palabras clave: Revista Historia de la Educación Latinoamericana, América Latina, educación virtual, universidad, reforma educativa, educación a distancia.

\section{OS CAMINHOS DAS REFORMAS PARA A VIRTUALIZAÇÃO UNIVERSITÁRIA NA AMÉRICA LATINA}

\section{RESUMO}

No presente artigo se analisa o crescente processo de incorporação de tecnologias de comunicação e informação na educação e de como está impactando os modelos semipresenciais previamente existentes. São analisadas, como derivado de seu impacto, as dinâmicas universitárias que estão inseridas em forte processo

\footnotetext{
Posdoctorado (UNESR, UNICAMP, UFF). Doctor en Educación (UNESR). Doctor en Derecho (UBA). Actualmente se encuentra vinculado en la Universidad de la Empresa (UDE). Economista (UCV), Magíster en Educación (UJMV). Ex Director del Instituto Internacional de la UNESCO para la Educación Superior en América Latina y el Caribe (IESALC). Decano de la Facultad de Ciencias Empresariales (UDE). Investigador activo del SNI del Uruguay.
} 
a typology of higher education institutions responding ways towards ICT such as re-engineering of online and part time institutions, new offers of college education $100 \%$ online, become special subjects of university's education into online programs and so as online management information trend. Thorough, a comparative analysis we developed a hypothesis of a new and slow online education and vanishing common space between new online education vs. traditional full time education.

Key words: History of Latin American Education Journal, Latin America, digital education, university, education changing movements, online education. de virtualização. É formulada a hipótese de uma tipologia dos caminhos, destacando-se a re-engenharia das instituições semipresenciais de educação à distância; as novas ofertas de educação presencial e a informatização da gestão administrativa. A partir de uma análise comparativa, se formula a hipótese da conformação de uma lenta educação digital e a desaparição das tradicionais distâncias entre uma educação à distância e uma educação presencial.

Palavras-chave: Revista História da Educação Latinoamericana, América Latina, educação virtual, universidade, reforma educativa, educação à distância.

\section{INTRODUCCIÓN}

La discusión sobre la tipología y las modalidades de la educación a distancia constituye una de las líneas de la investigación académica que propende además a visualizar los caminos y las prospectivas de la educación en tanto dicho proceso ha estado marcado por la incorporación de tecnologías pedagógicas en su sentido más amplio. Actualmente América Latina está avanzando diferenciadamente en los procesos de digitalización y virtualización de la educación superior pero se constata globalmente la convergencia tecnológica y la lenta desaparición de la dualidad de modalidades, como resultado de los diversos caminos de la virtualización y digitalización, y la consolidación de una dinámica evolutiva de parámetros comunes más allá de sus especificidades.

\section{La aparición de la Educación Superior a Distancia y la creación de modelos semipresenciales en la región}

En los setenta en la región y a escala mundial, se produjo un cambio significativo en la educación a distancia, al iniciarse su uso intensivo como metodologías de enseñanza también para el nivel superior. Ello se inició a través de un modelo unimodal semipresencial público. La educación superior a distancia asumió la forma de una educación semipresencial con el apoyo de materiales didácticos escritos, la existencia de tutores con una atención a través de sedes distribuidas en el país y en las cuales además se realizan las evaluaciones de los aprendizajes, ya que los exámenes se estructuraban para su realización presencial. Bajo este modelo educativo, 
el acceso no requirió de pruebas selectivas como en las universidades presenciales. El público estudiantil era en general de los excluidos del acceso público, de adultos y provenientes de sectores de menos ingresos económicos y capitales culturales, los cuales no lograban acceder al modelo de tipo dual dividido por un sector público con examen de ingreso y uno privado con matrícula arancelada.

En los noventa, un nuevo escenario se planteó en la educación a distancia a partir de la irrupción de nuevas tecnologías de comunicación e información que permitían la educación virtual y nuevas modalidades, así como una combinación cada vez más compleja y diversas de modalidades. Al tiempo, en los noventa, la diferenciación institucional que se había producido con la expansión de la educación privada, comenzó a mostrar una presencia cada vez más vigorosa de la oferta privada bajo esta modalidad. El ingreso de la bimodalidad, sin embargo se produjo a través de una alta diversidad de expresiones, en función de las particularidades propias de cada país. Una de las expresiones de esta nueva realidad, y sin duda la más conocida, fue la creación de nuevas universidades públicas, orientadas a ofertar bajo estas modalidades pedagógicas a distancia mediante modelos educativos semipresenciales, como la Universidad Estatal a Distancia (UNED) de Costa Rica, la Universidad Nacional Abierta (UNA) de Venezuela y la Universidad Nacional Abierta y a Distancia (UNAD) de Colombia. En estos países, estas instituciones nacieron asociadas al establecimiento de exámenes de ingresos selectivos en las universidades y funcionaron como ofertas de estudios terciarios sin significativas restricciones de ingreso. Mientras que las universidades públicas introducían restricciones al ingreso, se abrían en paralelo oportunidades de acceso abierto a menos costos por alumno. La base de su origen radicó en la Open University no tenía restricciones de ingreso sino de egreso, a partir de crecientes estándares de calidad en el proceso de aprendizaje con un seguimiento individualizado.

En la región estas modalidades de ofertas de educación a distancia nacieron además con menos niveles de autonomía, más dependientes de los Ministerios de Educación, y con presupuestos por alumnos significativamente inferiores que las restantes instituciones públicas. En Venezuela la UNA para 2004 tiene un presupuesto por alumno de 1.14 millones de bolívares, mientras que la universidad más costosa, la Simón Bolívar, tenía un 
costo por alumno de 12,89 millones de bolívares con una media nacional del sector público de 5,39 millones de bolívares. En el caso de Colombia, el costo por alumno de la UNAD para 2006 fue de 0,626 millones de pesos, mientras que la universidad más costosa, la Universidad Nacional tuvo un costo por alumno de 11,494 millones de pesos, con una media nacional de costo por alumno en la educación superior pública de 5,007 millones de pesos. Así, mientras que en Venezuela la diferencia entre la más cara y la UNA es 11,30 veces en Colombia, la diferencia alcanzó a 21,12 veces más ${ }^{2}$. Tales diferencias de recursos derivan en distintos niveles de calidad y de formación de sus docentes, así como de infraestructuras diferenciadas. Tales modelos también implicaron menor autonomía de las instituciones a distancia, lo cual marcó el recorrido político de estas tres universidades ha sido también el de la lucha por la obtención de la autonomía, que la UNED obtuvo a los cinco años de su creación, la UNAD relativamente en 2005 y que la UNA carece al estar regida por la Ley de Universidades de 1971 que establece una menor autonomía para las universidades experimentales.

Las nuevas demandas de acceso a la educación superior no presencial se expresaron tanto a través de estas nuevas instituciones orientadas a la oferta de modalidades educativas a distancia, como también en ofertas a distancia dentro de instituciones presenciales. En el primer caso se desarrollaron instituciones fundamentalmente públicas que nacieron con la exclusiva misión de desarrollar ofertas bajo la modalidad a distancia, y en el segundo caso, la expansión de la educación a distancia, se expresó en la conformación de modelos bimodales de educación superior al interior de las instituciones que en diversa proporción utilizaban y mezclaban ofertas presenciales y ofertas a distancia, tanto públicas como privadas.

Casos llamativos de bimodalidad se dieron por ejemplo en México (UNAM), Honduras (UNAH), y Argentina (UBA) donde se desarrollaron ofertas de educación a distancia al interior de las universidades autónomas, que comenzaron a estructurar un modelo bimodal en términos pedagógico, organizativo y tecnológico. Casi todas las grandes universidades autónomas de México, como la UNAM, la Universidad de Guadalajara, la de

2 Para Venezuela: Universidad Nacional Abierta. Plan Estratégico, Caracas, 2005. Para Colombia Sistema Nacional de Información de la Educación Superior (SNIES) - Observatorio de la Universidad Colombiana, 2008. 
Veracruz o la de Puebla, comenzaron a ofertar, en el marco de sus políticas de regionalización y de restricciones de acceso abierto, una nueva oferta educativa a través de la educación a distancia, bajo dinámicas semipresenciales con apoyo del libro y de guías didácticas especiales. La UNAM, como en muchos otros temas, abrió un camino en la región, al establecer en forma autónoma, al tiempo que la prueba selectiva de acceso bajo la gratuidad, una oferta educativa semipresencial sobre la base de propender a ofrecer los mismos niveles y estándares de calidad que la educación presencial universitaria.

En 1974 se crea el Sistema de Universidad Abierta (SUA) de la UNAM que propendía a ofrecer oportunidades de acceso a la institución, dado el establecimiento de cupos y exámenes a la educación presencial, para aquellos que no lograban ingresar. El modelo del SUA posteriormente también se basó en una selectividad de acceso, pero con menor presión de postulantes versus ingresantes. La Universidad Central de Venezuela (UCV) también desde la década del setenta, asociado al establecimiento de restricciones de acceso, propendió, aunque en muy reducida proporción, a desarrollar modalidades de educación a distancia para quienes no podían acceder a los sistemas presenciales. A diferencia otro modelo de expansión de la educación a distancia al interior de las universidades públicas se dio, en Honduras, tanto en la Universidad Nacional Autónoma de Honduras (UNAH) desde 1985, como posteriormente en la Universidad Nacional Pedagógica (UNPFM). En estas instituciones se conformó un relativo modelo bimodal con una educación presencial por una parte y por la otra, una educación superior a distancia (semipresencial) acotada, y cuyas diferencias, en teoría eran solo de metodología de enseñanza y no por la forma del acceso, ya que se carecía de selectividad de acceso a la educación presencial. En este caso, la educación a distancia, se viabilizaba fundamentalmente para cubrir demandas en parte del territorio nacional en el cual no había sedes regionales de esas instituciones que ofertaran educación superior en forma presencial. Ambos modelos con el tiempo, aún siendo de acceso libres y sin sistemas de evaluación, se tendieron a diferenciar en la calidad, el tipo de estudiante y el tipo de ofertas disciplinarias ${ }^{3}$.

\footnotetext{
3 Algunas de las diversas situaciones a nivel nacional se pueden ver en Claudio Rama y José Pardo (editores), $L a$ educación superior a distancia: miradas diversas desde Iberoamérica (Caracas, 2010) http://observatoriouniversitario.info
} 
En otras universidades autónomas se desarrollaron modalidades bimodales pero asociadas a las propias facultades y escuelas, y no como una modalidad educativa distinta a nivel de toda la institución sino a nivel de las unidades académicas de base, de las carreras o materias. Ellas se asociaron al nombre de "estudios libres" y tuvo sus ejemplos en la Universidad de Buenos Aires (así como varias de las grandes públicas de Argentina) y en la Universidad de la República en el Uruguay (UDELAR). Ellas, dados sus modelos de acceso abierto y la saturación de las aulas ante las limitaciones físicas de espacio, establecieron modalidades de educación a distancia de tipo semipresencial, donde algunos estudiantes estaban autorizados u obligados a llevar asignaturas semipresenciales y solo estaba obligados a rendir en forma presencial los exámenes finales o parciales junto a aquellos que seguían el curso en forma regular. Estos modelos sin embargo tuvieron altos niveles de deserción ${ }^{4}$. El carácter fragmentado de las universidades públicas con fuerte peso de las facultades facilitó el establecimiento de estas modalidades de oferta a distancia y de sus formas bimodales, no a nivel central, sino independientemente a nivel de las distintas unidades académicas dadas las diferenciadas presiones de cobertura y de problemas locativos.

\section{Tabla 1. Formas bimodales de la Educación Superior a distancia semipresencial}

\begin{tabular}{|l|l|l|}
\hline Sistémicas & $\begin{array}{l}\text { Creación de instituciones dedicadas a ofertar ex- } \\
\text { clusivamente bajo la modalidad de educación a } \\
\text { distancia. }\end{array}$ & $\begin{array}{l}\text { Venezuela (UNA), Costa Rica } \\
\text { (UNED), Colombia (UNAD), } \\
\text { Ecuador, (UTPL), México (TEC) }\end{array}$ \\
\hline Intrauniversitarias & $\begin{array}{l}\text { Creación de unidades de la universidad a nivel } \\
\text { central dedicadas exclusivamente a ofertar edu- } \\
\text { cación a distancia. }\end{array}$ & $\begin{array}{l}\text { México (UNAM, UDG, Veracruz, } \\
\text { Honduras (UNAH, UNPFM) }\end{array}$ \\
\hline Interfacultades & $\begin{array}{l}\text { Separación de estudios regulares y de estudios } \\
\text { libres, a nivel de las carreras, y desarrollo de mo- } \\
\text { dalidades de educación a distancia a nivel de la } \\
\text { facultad o carrera }\end{array}$ & $\begin{array}{l}\text { Uruguay (UDA), U. de Córdoba, U. de la } \\
\text { (UBa) }\end{array}$ \\
\hline
\end{tabular}

Fuente: Elaboración del autor

\footnotetext{
4 Ver al respecto los diversos estudios de casos nacionales en Ángel Hernández, et al., (editores) La deserción en las instituciones de Educación a Distancia en América Latina y el Caribe (República Dominicana: UAPA, 2009). http://observatoriouniversitario.info
} 
En general (salvo algunas excepciones como Honduras, Argentina o Uruguay que de hecho funcionaron como modelos bimodales sin restricciones de acceso, la educación superior a distancia), durante esta fase se estructuró bajo la existencia de selección de acceso presencial por un lado, y acceso abierto para un público de bajo capital cultural y de bajos ingresos económicos, por el otro. Ello marcó el carácter gratuito y público de la educación superior a distancia y su propio rol como una modalidad educativa de menor calidad durante esta fase. Sin embargo, también se comenzaron a desarrollar en la región en esta fase, algunas manifestaciones de ofertas de educación a distancia por parte de instituciones privadas.

Entre esos casos cabe destacar la Universidad Técnica Particular de Loja en Ecuador y el Tecnológico de Monterrey en México, las cuales en sus inicios desarrollaron también un modelo educativo semipresencial y se expandieron en sus respectivos países que carecían de amplios sistemas de acceso públicos a distancia. Ambos siguieron orientaciones inversas. El Tecnológico de Monterrey, institución sin fines de lucro nacida en el año 1943 y muy vinculado a sectores empresariales de México y que desde sus inicios propendió a ofertas de calidad, se focalizó en estudiantes de altos ingresos bajo un modelo educativo basado en la $2^{a}$ generación de la educación a distancia con uso intensivo de videoconferencias ${ }^{5}$. Tal modelo fracasó por las desconfianzas a la enseñanza virtual y por cuanto las familias no estaban dispuestas a pagar altos costos por una educación que era a distancia y de imagen de inferior calidad. El rol educativo de lo presencial no pudo ser solucionado por una educación virtual pero dentro de los campos universitarios. El Tecnológico de Monterrey (TEC) produjo un cambio radical y se reorientó a un modelo presencial de élites con alta incorporación de tecnologías de comunicación e información. Recién posteriormente con la aparición de las tecnologías digitales avanzó hacia la creación de un modelo adicional de educación superior a distancia virtual, que asumió el nombre de TEC Virtual y de TEC Milenium para sectores de menos ingresos.

Otra iniciativa privada significativa en términos de su presencia activa en los ámbitos académicos, es la Universidad Técnica Privada de Loja (UTPL), de la ciudad de Loja en el interior de Ecuador, que desarrolló un

En los noventa el TEC de Monterrey se internacionalizó en la región con un modelo de videoconferencia altamente costoso que fue posteriormente abandonado. Inclusive en México, abandono la oferta a distancia para 
modelo tradicional de educación superior a distancia semipresencial con apoyo en sedes y focalizado en sectores de menores ingresos, de difícil accesibilidad a la educación presencial y con bajos costos de la matrícula. En este caso la viabilidad económica estuvo dada por su gran escala, los bajos costos salariales en el interior del Ecuador, el carácter no lucrativo religioso de la institución, una eficiente gestión y controles de calidad y un sistema de cofinanciación dado por un aporte financiero público a algunas instituciones privadas en el país.

Desde de la década del ochenta, la educación superior a distancia se fue expandiendo lentamente en toda la región, aún a pesar de las múltiples restricciones políticas, académicas y en algunos casos inclusive normativas $^{6}$. La expansión de esta modalidad educativa en general se dio sobre la base de ausencia de políticas públicas, y más aún, de un cierto ocultamiento de esta realidad educativa. Salvo los casos de la UNED, la UNAD y la UNA que siempre tuvieron una mayor visibilidad y se constituyeron como modelos de política pública por ser universidades, el resto de las ofertas públicas no estaban claramente develadas en las estadísticas, permitiendo así un cierto ocultamiento de una realidad en lento pero persistente crecimiento. La expansión de este modelo pedagógico a distancia, carente de tecnologías interactivas y que asumió la forma de educación semipresencial abierta aumentó con el ingreso hacia fines de los noventa de nuevos proveedores locales como fueron por ejemplo la Universidad del Caribe (UC) y la Universidad Abierta para Adultos (UAPA) en República Dominicana, la Universidad de Salta y la Universidad Blas Pascal en Argentina, ALAS Peruanas y ULADECH en Perú, la Universidad de Bucaramanga en Colombia, la Universidad de Paraná en Brasil o la Universidad Rafael Belloso Chacín en Venezuela que ingresaron con los mismos modelo de educación a distancia semipresencial abierta. Lo interesante es que la mayor parte de ellas son universidades gestadas desde el interior de sus respectivos países. La incapacidad de crecer bajo modelos presenciales en mercados pequeños llevó a estas iniciativas a encontrar en la oferta a distancia, el mecanismo para alcanzar mayores escalas.

sectores de altos ingresos y se concentró en una oferta bimodal de un modelo presencial costoso y un modelo virtual de menos costo, expresado en instituciones diferenciadas como son el TEC Milenium y la Universidad Virtual del Sistema TEC.

6 Patricia Torres y Claudio Rama (editores), La educación a distancia en América Latina. Realidades y Tendencias. (Brasil: 2009). Los diversos estudios nacionales se pueden ver en http://observatoriouniversitario.info 


\section{El impacto de las nuevas tecnologías y los nuevos caminos de la educación a distancia}

Una nueva fase de la educación a distancia se abrió en América Latina con la irrupción de las nuevas tecnologías digitales de comunicación e información desde fines de la década del noventa. Ellas plantearon el inicio de una nueva generación de la educación a distancia y la transformación del modelo educativo semipresencial que ha dominado en la región desde los inicios. Este proceso de incorporación de lo digital en la oferta semipresencial se ha expresado en diversas reingenierías en las instituciones de educación a distancia en la región 7 . La aparición del nuevo escenario tecnológico ha planteado una nueva generación de la educación superior a distancia, que viabiliza además el pasaje desde el uso de un instrumento exclusivo de comunicación a una amplia paleta de utilización de las diversas tecnologías, de un modelo puro de educación a distancia a un modelo híbrido, que combina elementos abiertos (libros), analógicos (medios hertzianos), digitales (Internet), sobre la base de la propia convergencia que permite lo digital.

El escenario tecnológico está impulsando que las instituciones que brindaban ofertas de educación, y entre ellas también las a distancia, estén abocadas en los últimos años la incorporación de esas tecnologías derivadas de la revolución de la microelectrónica. Es una transición, que en algunos casos de la educación a distancia, asume formas de reingenierías organizacionales, dadas por el pasaje desde tecnologías analógicas a tecnologías digitales, desde modalidades de comunicación unívocas hacia modalidades de comunicación biunívocas, desde soportes hertzianos con baja segmentación, alta cobertura pero que estaban acotadas a las fronteras nacionales dadas las características de las autorizaciones de potencia y altura de torre de los medios de esos medios de comunicación hacia mecanismos y modalidades de comunicación a través de las redes digitales no carecen de límites geográficos de cobertura y que por ende en este caso tales reingenierías pueden incluir dinámicas internacionales. Desde materiales instruccionales planos hacia procesos de aprendizaje en red que permiten el hipertexto como mecanismo de aprendizaje y de los modelos de simulación digitales que se articulan a las concepciones más modernas sobre la experimentación como mecanismos de aprendizajes más eficientes.

Claudio Rama, Las reingenierías de las universidades a distancia en América Latina (República Dominicana: Ediciones UNICARIBE - UDUAL, 2011). 
La irrupción de lo digital en los noventa ha planteado tanto transformaciones en las instituciones que tenían ofertas a distancia como un incentivo al ingreso de nuevos proveedores, tanto locales como internacionales, ante el abaratamiento de los costos y los requerimientos de alcanzar nuevas escalas. En el caso de las tradicionales universidades a distancia ello se expresó en el pasaje desde las tecnologías de la primera y la segunda generación hacia instituciones basadas en tecnologías de tercera generación y que, por la convergencia, pueden integrar a las modalidades tecnológicas anteriores en sus plataformas digitales. Esta modalidad además facilita tanto formas puras como híbridas dadas por las diversas combinaciones posibles de tecnologías de comunicación (presencial, libro, televisión, etc.) en el proceso de enseñanza.

Las tecnologías digitales están viabilizando con distinta intensidad estas reingenierías de proceso, de producto y organizacionales, como parte de la reforma de la virtualización de la enseñanza. El nuevo escenario implica una tecnología exclusiva como también una amplia paleta de utilización tanto de las viejas como de las nuevas tecnologías, ya que se abren nuevas modalidades de ofertas al interior de la educación a distancia, complejizando la propia bimodalidad, ya no solo puede presencial versus semipresencial abierta, sino también de una educación a distancia abierta versus una educación digital. Sin embargo, a pesar de la diversidad, el eje articulador es lo digital.

La transformación de las ofertas de educación a distancia desde aquellas basadas en la utilización de didácticas y pedagogías como el libro y el tutor presencial, hacia las digitales encuentra su causalidad en el incremento de la calidad, en el aumento de la cobertura y en la disminución de los costos por alumno que el nuevo modelo significa. El eje de la incorporación de productos e industrias culturales (libro, disco, video, internet o software) está asociado a los beneficios educativos en la enseñanza. Las reingenierías que se introducen para facilitar e impulsar los cambios tecnológicos, no son reformas dentro de un mismo paradigma, sino buscando introducir cambios en la organización educativa para viabilizar el desarrollo de nuevos procesos y productos. Como referimos, las reformas derivadas de esas reingenierías se asocian a mejorías en la calidad, en la cobertura y en los costos. Las nuevas situaciones dadas entre otros por menores costos para 
los usuarios, recursos didácticos más fieles a la realidad, miniaturización de dichos recursos o mayor interactividad, refuerzan las líneas anteriores de costos, calidad y cobertura, contribuyendo a un ciclo continuo de digitalización y de expansión de esta modalidad educativa. Esos impactos serían como se refiere a continuación:

a. La calidad se incrementa dada la mayor capacidad de interacción en tiempo real o diferido en la lógica enseñanza - aprendizaje, por la capacidad de utilizar con la convergencia tecnológica de una mayor cantidad de materiales instruccionales (vídeos, láminas de power point, imágenes planas, mapas mentales, aplicaciones, audio), por la posibilidad de acceder a materiales interactivos, por el mayor peso en el autoaprendizaje y el incentivo a enfoques constructivistas. La calidad, derivada de la confluencia de medios visuales, auditivos y escritos, y la interacción y la experimentación, aumentan la retención y la comprensión de los temas.

b. La cobertura por su parte se puede incrementar significativamente, al incorporarse otros sectores sociales dotados de mayor conectividad y capacidad de absorber esos costos y los de los equipamientos, así como estudiantes localizados en el extranjero dado el acceso en red o con movilidad reducida como las personas con discapacidades o privadas de libertad. En términos de cobertura la incorporación de tecnologías digitales permite una ampliación de la cobertura asociado a segmentaciones y escalas reducidas.

c. Los costos finalmente se articulan en una ecuación de costos - calidad - cobertura distinta a la existente en la educación presencial, imponiendo una estructura con menores costos continuos por alumno, dada la caída de los costos de los envases de casetes y libros, así como de sedes y aulas para realizar las asistencias y tutorías, y la transferencia de parte de los costos de conexión y de los equipamientos a los estudiantes. Igualmente los costos caen por las diferentes competencias y de salarios entre los docentes presenciales y los tutores digitales, así como por el abaratamiento continuo de los costos de conectividad y equipamiento digital. Los materiales instruccionales tienen una durabilidad mayor así como una mayor capacidad de actualización. Igualmente, 
ya existe en creciente mercado de compra e intercambio de cursos digitales que abarata el costo de los materiales al amortizarlos entre más estudiantes. Actualmente se ha pasado a diversidad de recursos de aprendizaje, abandonando el texto único especialmente realizado y diseñado. Muchas ofertas además también se apoyan en libros de texto comerciales que tienen estándares de calidad superiores.

Estas y otras causas más como la nueva competencia interuniversitaria, están incidiendo en la expansión de la oferta no presencial y en que las ofertas de educación superior a distancia, formadas desde los años setenta, estén lentamente avanzando hacia su transformación al incorporar tecnologías digitales. Tales transformaciones no se darán en todas las instituciones, ya que el pasaje de un modelo semipresencial abierto a uno virtual, tanto semipresencial como puro, si bien por un lado atrae nuevos estudiantes, por el otro expulsa aquellos de bajos recursos localizados en zonas y hogares de baja conectividad y recursos económicos. Ello impone en algunos casos, el no abandono de los modelos educativos semipresenciales, sino la incorporación de una nueva bimodalidad al interior de la educación a distancia.

Sin embargo, tanto el pasaje de un modelo de educación a distancia a otro, como la sola introducción del nuevo modelo virtual, deriva en una reingeniería en las instituciones y en sus modelos educativos, que para su logro deben articularse a través de una planificación estratégica que permita introducir las amplias transformaciones que se requieren ya que ellas implican cambios en las competencias y actividades de los docentes y funcionarios, en el tipo de estudiante, en los procesos de aprendizaje, en los modelos de gestión, en las inversiones económicas y tecnológicas, y en las estructuras organizativas internas de la universidad.

La transformación de las instituciones de educación superior a distancia a partir de la utilización de las tecnologías digitales, implican también aumento de costos globales y el riesgo derivado de la posible pérdida de alumnos de las modalidades semipresenciales abiertas, de los costos políticos y económicos de la desarticulación de las redes de sedes presenciales, de los requerimientos iniciales y futuros de inversiones en infraestructura tecnológica, y de competencias superiores de los equipos administrativos 
y docentes, así como en los derivados de la digitalización de los recursos didácticos y de los derechos intelectuales de estos.

En términos de cobertura, se logra una expansión a nuevos sectores de mayores ingresos económicos y tal vez a mayores demandas diversificadas de campos de conocimientos y poder ofrecer una mayor cantidad de cursos que los modelos de educación abierta. Igualmente la incorporación de las tecnologías digitales, contribuye a normalizar y simplificar los procesos administrativos y abaratar los costos fijos por alumnos. Las escalas superiores al tiempo facilitan la concentración de la matrícula y menores costos por estudiante. Inversamente, las políticas públicas aumentan las exigencias y contrapesan con mayores costos estas mayores oportunidades económicas.

Al tiempo, el nuevo escenario tecnológico, y por ende las nuevas estructuras de costos, dadas también las nuevas demandas de acceso por parte de sectores de mayores ingresos económicos, han planteado la posible irrupción de nuevos proveedores de educación superior a través de la educación a distancia los cuales en general tienden a ser privados. Salvo en el caso de México que se han creado varias universidades estatales virtuales, una universidad a distancia nacional y Brasil, en el resto de la región no ha habido iniciativas significativas públicas en la materia. La apertura a proveedores nuevos privados no se ha expresado en la habilitación de proveedores internacionales, sino que en varios casos se han planteado claras limitaciones normativas. Sin embargo, más allá de esto, hay un mayor peso privado que antes en la oferta a distancia. Mientras que en la primera ola de la educación superior a distancia en los setenta y ochenta la presencia pública era dominante y todas eran ofertas locales, en el actual escenario, hay una preponderancia de oferta privada, junto con un aumento de la presencia de ofertas con alianzas o directamente extranjeras, asociadas al aumento en la utilización de tecnologías digitales.

Igualmente en el nuevo contexto se constata una mayor regulación pública así como una mayor diversidad de los modelos educativos al interior de la educación a distancia. En esta nueva fase se verifica cómo la región ha ido pasando muy rápidamente de modalidades homogéneas y monopólicas de ofertas de educación a distancia a través de las instituciones públicas de educación a distancia creadas en los setenta y en los ochenta, 
hacia un amplio, complejo y diverso escenario institucional y pedagógico, que se está expresando en un aumento significativo de la cobertura de la educación superior a distancia en la región. Ello se está dando en el marco de un nuevo proceso de diferenciación institucional tanto a nivel del sector público como privado asociado a nuevas demandas y a diferenciación de modelos educativos, que incentivan una tendencia a la despresencialización de la educación superior.

En este proceso visualizamos varios caminos. Por una parte la irrupción de nuevas ofertas de educación superior a distancia, por el otro la transformación de las ofertas existentes de educación superior a distancia a partir de la incorporación diferenciada de las tecnologías digitales y, en algunos casos, derivado de reingenierías institucionales, el desarrollo de nuevos modelos educativos. Igualmente, hay una creciente incorporación de dinámicas de enseñanza virtuales o digitales en la enseñanza presencial.

Una de estas manifestaciones en el nuevo contexto de tecnologías y demandas sociales es la nueva oleada de instituciones de educación superior a distancia, fundamentalmente públicas en varios países. Estas nuevas ofertas se desarrollaron como mecanismos para aumentar la cobertura a menos costos, en las cuales se desarrollan una variedad de modelos educativos. En algunos casos se reafirma el modelo abierto semipresencial pero sobre la base de una utilización de tecnologías virtuales. Los casos más significativos de política pública recientes han sido la Universidad Abierta de Brasil, los Centros Regionales de Educación Superior (CERES) en Colombia y la municipalización de la educación superior en Cuba. Ellos expresan modalidades diferenciadas de aumento de la cobertura ya no presencial, de acción pública directa y de articulación entre el Estado y las diversas demandas de acceso. Son además modalidades de acceso libre sin exámenes.

La otra orientación se focaliza en las complejas reingenierías de la educación superior a distancia que están desarrollando la mayor parte de las instituciones con programas de educación sobre la base de las tecnologías de la primera generación (libro y guías didácticas) o de la segunda (radio, telefonía o televisión), hacia nuevas modalidades que en distinta proporción van incorporando los nuevos ejes tecnológicos. 
La tercera, más compleja y con baja información, refiere a la incorporación de las pedagogías informáticas en el aula y la lenta digitalización de la interacción estudiante docente, entre estudiantes, y entre estudiantes y recursos instruccionales. En tal sentido podemos visualizar como caminos de la virtualización universitaria en América Latina a la virtualización de la educación semipresencial preexistente a partir de reingenierías tecnológicas y pedagógicas, la irrupción de nuevas ofertas universitarias totalmente virtuales, la aparición de proveedores transfronterizos internacionales, la incorporación de pedagogías informáticas en la educación presencial, la informatización de la gestión académica y administrativa de las instituciones educativas, y la digitalización de los recursos didácticos.

\section{La reingeniería de las instituciones semipresenciales de Educación a Distancia}

En América Latina se inició el camino de la educación a distancia a nivel universitario desde fines de los setenta cuando se crearon instituciones y programas públicos de acceso a través de modelos semipresenciales. A diferencia de otras partes del mundo, la región carecía de una experiencia histórica significativa de educación a distancia en el sector universitario o en los otros niveles. A partir de entonces, y muy puntualmente en algunos países, al calor de la expansión de las demandas de acceso y el establecimiento de cupos de acceso, y de las influencias de la Open University de Inglaterra y de la UNED de España que fueron centros de referencia y de asistencia técnica, se comenzaron a instalar ofertas bajo esta modalidad en Colombia, Venezuela, Costa Rica y México. La educación a distancia no incorporó señales de radio o de televisión, salvo Brasil, e impuso un modelo semipresencial nacional con apoyo de recursos didácticos impresos. Este modelo de baja calidad también tuvo poca cobertura. Se expandió en esos pocos países a través de instituciones públicas que propendían ofrecer un acceso a quienes no alcanzaban a ingresar a los selectivos cupos públicos gratuitos o a los crecientes pagantes ingresos privados.

Desde fines de los noventa con la digitalización se comenzó lentamente a introducir cambios en los modelos pedagógicos, tanto presenciales como semipresenciales. Las nuevas tecnologías, aunque tardíamente, facilitaron tanto reestructuras e reingenierías en las tradicionales ofertas a 
distancia, como también el ingreso de nuevas instituciones a distancia, incorporando plataformas digitales para apoyar las ofertas a distancia. En el marco de estas reingenierías se pasó en muchos casos de modelos semipresenciales a un "blended learning" o educación semivirtual, siempre ajustado a los marcos normativos dominantes que limitan la total virtualización. La plataforma de fuente abierta Moodle constituyó una verdadera revolución que permitió esas reingenierías de la educación a distancia por su incidencia en los costos. Así, una de las vertientes más significativas de la virtualización universitaria en la región se llevó a cabo, lentamente, al interior de las tradicionales instituciones de educación a distancia a través de reingenierías educativas que transformaron su modelo de enseñanza aprendizaje. Algunas instituciones como la UNED (Costa Rica) resolvieron una estrategia de virtualización unimodal. En esta, la transición a la digitalización fue total, y se pasó de un formato semipresencial a un formato semi-virtual, incorporando plataformas virtuales, tutores virtuales y recursos didácticos digitalizados, fundamentalmente a través de Moodle. Otras, dado las resistencias al cambio y las características de los estudiantes y sus entornos, estructuraron una dinámica bimodal y se desarrolló un acceso a los estudiantes bajo ambas modalidades, tanto semipresencial como semivirtual.

En este contexto, las tradicionales instituciones a distancia que caracterizaron históricamente el panorama latinoamericano como la UNED (Costa Rica), UNA (Venezuela), UNAD (Colombia), SUA (UNAM-México), UTPL (Ecuador) y TEC (México), o bajo la educación abierta típica de algunas universidades públicas de masas (UBA - Argentina), han pasado o están en transición con diversa intensidad hacia modelos semivirtuales. Algunas de las instituciones como la UNAD que facilitaron la libre selección por los estudiantes entre la modalidad semipresencial y semivirtual, permiten constatar cómo sistemáticamente todos los años aumenta la inscripción a la modalidad semivirtual, mostrando el traslado de la demanda estudiantil ante condiciones iguales de exigencias. En otros casos donde la dualidad se plantea entre la modalidad semipresencial y la modalidad virtual, como la UNAH (Honduras), se verifica que los estudiantes optan por el sistema semipresencial en tanto es menos exigente y riguroso que el virtual o el mismo presencial. 
En otro caso, en la UNAM (México) que tiene tanto una oferta semipresencial tradicional como una nueva oferta virtual, ambas con accesos selectivos competitivos, también se constata el crecimiento continuo de la demanda de oferta virtual. Ambas tienen los mismos niveles de calidad y actúan sobre grupos sociales o condiciones personales distintas, pero en tanto la brecha digital se va reduciendo, se van trasladando estudiantes a un acceso bajo una modalidad de enseñanza virtual.

La expansión de la modalidad virtual sin embargo, no ha sido mayor en la región por los marcos normativos que imponen el modelo semipresencial, así como por las limitaciones de conectividad o el tipo de estudiantes, aún cuando estas dos restricciones se han ido reduciendo muy rápidamente al generalizarse el acceso a la cultura digital. Van quedando entonces restricciones normativas en parte asociadas a los viejos paradigmas y a los propios temores de algunos sectores de la comunidad académica por los cambios que esos procesos implican. Ello limita la oferta totalmente virtual en la mayoría de los países de la región como por ejemplo en Brasil, Argentina, Ecuador, Chile que no permiten ofertas totalmente virtuales. Los casos analizados en México (UNAM) y en Colombia (UNAD), muestran el claro corrimiento de las demandas de acceso hacia un suministro virtual, y como ello contribuye a la digitalización al interior de las instituciones donde un rol destacado los tienen los propios tutores virtuales que presionan hacia la informatización pedagógica. También son de destacar las limitaciones a la expansión virtual en países donde tanto ella como la oferta semipresencial no están autorizadas para el sector privado como en Uruguay o Bolivia.

En general en este tema, se mantuvo un modelo semipresencial, pero virtualizado al agregarse recursos instruccionales digitales, interacción en plataformas y entrega de trabajos y evaluaciones de aprendizaje en las aulas virtuales, como estrategia para aumentar la calidad de los procesos de enseñanza, más allá de la preponderancia de una baja interacción y de la existencia de recursos didácticos planos.

\section{Las nuevas ofertas de Educación Universitaria 100\% virtuales}

En la región prácticamente se carece de educación totalmente virtual. Esta modalidad de enseñanza mediada en su totalidad por ámbitos digita- 
les se utiliza dominantemente en el sector informal en certificaciones no reguladas de educación continua. Su oferta en el sector formal o regulado, recién se está comenzando a iniciar gracias a una relativa flexibilización de las tradicionales políticas públicas restrictivas. Ello se está produciendo en México, Colombia y Venezuela que han comenzado a habilitar ofertas universitarias totalmente virtuales, tanto por parte del sector público como privado. También en Puerto Rico en tanto se regula por las normas de los Estados Unidos donde existen dichas modalidades ampliamente.

Sin embargo, la flexibilización para habilitar ofertas locales virtuales, no significa una habilitación del acceso a nuevos proveedores internacionales, sino que ello requiere el licenciamiento previo, en algunos casos hasta para ofertar en el país, para tener el reconocimiento oficial a las certificaciones. En los casos que se produce fuera del país el proceso de enseñanza - aprendizaje, ella requiere igualmente revalida u homologación con sus propias complejidades. El Caribe es la única zona donde la oferta transfronteriza es ampliamente reconocida y autorizada. En la región continental a diferencia, hay una relativa tendencia a incorporar limitaciones, incluyendo la obligatoriedad de asociación con una institución local como en El Salvador.

A la fecha existen apenas unas pocas universidades exclusivamente virtuales, siendo todas muy recientes. Ellas son fundamentalmente de México, como la Universidad Virtual de Guadalajara (es un brazo de la Universidad de Guadalajara), la Universidad Virtual de Estado de Guanajuato (pública), el Consorcio Clavijero del Estado de Veracruz (pública), la Universidad Virtual del Estado de Michoacán (pública), la Universidad Mexicana en Línea (UMEL), el Tec Virtual y la Universidad Fray Luca Paccioli (UFPL) (privada). También irrumpen, pero más lentamente en Colombia con la Universidad Católica del Norte (privada), en Panamá con Globalink Virtual University (privada, aún solo programas terciarios autorizados), en Venezuela relativamente algunos pocos programas de la Universidad Belloso Chacín y la Universidad Nacional Abierta y en Puerto Rico dentro del Sistema Universitario Ana G. Méndez - SUAGM (privada) a través del Programa de Educación a Distancia. Al interior de muchas universidades existen unidades especialmente dedicadas a la oferta virtual, pero carecen de la capacidad de ofrecer certificaciones universitarias completas virtuales, encarando algunos cursos virtuales dentro de los programas universitarios u ofertas de extensión y educación continua. 
La existencia de procesos de evaluación de los aprendizajes en Brasil (Examen Nacional de Aprendizaje - ENADE), en México (CENEVAL) o en Colombia (ICFES - Exámenes de Estado), facilitan indirectamente la propia expansión de los sistemas 100\% virtuales de enseñanza-aprendizaje. Ello por dos determinantes. Por un lado permiten evaluar en términos comparativos los resultados del aprendizaje de las diversas modalidades. Por otra parte, dan una garantía al ser mecanismos externos de evaluación de los aprendizajes. Los exámenes de Brasil, por ejemplo, han mostrado que los aprendizajes no son significativamente diferenciados, y que los costos educativos, aunque levemente, se han reducido ${ }^{8}$. Tal realidad ha planteado una nueva confrontación del modelo semipresencial frente al modelo virtual, en una dinámica donde se han continuado reduciendo las barreras a la virtualización, dada la verificación de la eficacia pedagógica de la educación virtual. Ello se constituye en un requisito fundamental, en tanto sin sistemas de evaluación iguales, no podrán superarse las fuertes resistencias y limitaciones. En este sentido, la convergencia digital solo se podrá dar y también la plena utilización de las tecnologías y procesos digitales, en sus diversas dimensiones gracias a la existencia de sistemas de evaluación externos de los aprendizajes.

Ya para 2010, en México, el 10\% de los estudiantes terciarios están inscritos en programas no presenciales y en Brasil, el 16\% de los nuevos estudiantes se inscriben en programas a distancia. Ambas modalidades, pero fundamentalmente la educación semipresencial con o sin apoyo digital, alcanzan a cubrir cerca de 1,5 millones de estudiantes universitarios en la región. Con 21 millones de estudiantes terciarios en este año, la llamada educación a distancia en sus diversas expresiones tradicionales representarían un nivel de élite alcanzando a cubrir un poco más del 7\% de la matrícula regional.

\section{La virtualización de segmentos puntuales de la Educación Presencial}

La tendencia más significativa de la digitalización de la educación superior se está dando a través en la incorporación de herramientas informáti-

8 Patricia Lupión y João Vianney, "La Educación a Distancia en el Brasil”, en La educación superior a distancia: Miradas diversas desde Iberoamérica, eds. Claudio Rama y José Pardo (Caracas: 2010), 15-44. 
cas en la educación presencial. Ello hace que la dimensión real del proceso de educación digital sea muy superior a ese 7\% referido, si incorporamos la compleja y diversa virtualización de la educación presencial, gestada a partir de la introducción de pedagogías informáticas, con dinámicas de enseñanza basadas en aplicaciones en laboratorios informáticos, acceso a recursos didácticos en la red, evaluaciones informatizadas, o directamente asignaturas de enseñanza virtuales.

Esta dinámica muestra una significativa transformación universitaria a través de la digitalización y la virtualización del proceso de enseñanza. Ello, más allá de la realización de los procesos de inscripción, de entrega de trabajos, de intercambio en la red, o de la existencia de páginas Web de las universidades, donde más allá de los distintos niveles de interacción, se aprecia un avance hacia interacciones más complejas y focalizadas tanto en el aprendizaje como en la gestión. Se calcula que cerca del 5\% del actual proceso de enseñanza está virtualizado, que daría cerca de un millón de estudiantes. Entre los dos sistemas, podemos decir que cerca del 10\% del tiempo de enseñanza del estudiante terciario en la región es a distancia, siendo el virtual la forma creciente, tanto bajo formatos a distancia como presenciales.

Aunque en sus inicios la educación virtual se concibió como una nueva generación en la educación a distancia, en el marco de un enfoque educativo que diferenciaba ambas modalidades, la presencial o la virtual, se constata crecientemente una diversidad de situaciones con múltiples articulaciones. Se podría concebir cómo se está pasando de una bimodalidad a una convergencia digital, donde, más allá de la dominante presencial o a distancia, hay una lógica virtual que se desarrolla en paralelo al proceso de convergencia digital.

La digitalización ha comenzado a reestructurar la tradicional educación presencial, como parte de un cambio mediante el cual la educación ha ido incorporando un enfoque por competencias. En este sentido, la pedagogía informática apunta a impulsar el enfoque curricular por competencias y un aprender haciendo. Con ello la dinámica educativa se está articulando a partir del uso de recursos digitales. Ello se expresa en la incorporación de ipod, ebook, laboratorios, aplicaciones informáticas, aulas virtuales, web 
2.0, proyectores de láminas como powert point, etc., en tanto todas ellas permiten mejor nivel de retensión y de adquisición de competencias. Las pedagogías informáticas facilitan además la transición desde aprendizajes lineales al hipertexto; de solo medio de apoyo (libro) a los multimedia; de medios sin interacción a medios interactivos; de microbibliotecas a Internet; de aprendizaje pasivos a autoaprendizajes sobre aplicaciones informáticas, con lo cual cambia el modelo educativo que se apoyaba en recursos didácticos como la tiza, la lengua y el pizarrón.

La computadora es el nuevo instrumento educativo, tal como el powert point o las pantallas interactivas están cambiando la dinámica de las clases presenciales e Internet cambia la forma de acceder a la información, en tanto formas de expresión de la digitalización en la enseñanza. El incremento de la cantidad de computadores por estudiante mide esa evolución. La pedagogía informática entre otras a través de conectividad para el acceso, interacción estudiantes - recursos instruccionales o estudiantes - profesores, o de autoaprendizaje a través de aplicaciones específicas, se transforman en las formas dominantes para construir las competencias. La informática se expande además ante las dificultades de reproducir la realidad en la práctica o la dificultad de supervisar educativamente las pasantías y las prácticas estudiantiles. En este sentido, los sistemas educativos están pasando de la valorización de la práctica presencial para alcanzar el aprendizaje, al uso de la informática para adquirir las competencias. En lo académico hay una demanda de calidad en términos de adquisición de competencias que se logran alcanzar con la pedagogía.

Sin duda, la pedagogía informática tiene limitaciones pero sus resultados en el aprendizaje, y especialmente en las asignaturas más profesionalizantes, son muy superiores en tanto permiten ingresar a una educación haciendo, no a practicar "de" la computadora, sino "con" la computadora. Obviamente es este un proceso que depende de los campos disciplinarios y de la complejidad de las herramientas informáticas, así como del propio desarrollo de las tecnologías. En su evolución estas superan los modelos de estímulo respuesta, el simple concepto de acceso a la información, o de

Silva Pontes, Edel Guilherrme, "Tecnologías en el aprendizaje de la estadística” (Tesis doctoral. Inédita, en Universidad de la Empresa, 2012), 328. Esta tesis evalúa el resultado de aprendizajes en estadística entre un grupo sin pedagogía informática y otro cuyo aprendizaje se realizó con herramientas informáticas y verifica cómo las pedagogías informáticas permiten aprendizajes significativos. 
interacción pasiva, para permitir evolucionar a herramientas de formación de competencias colaborativas con mayores niveles de interacción y representación de la realidad de los ámbitos del trabajo. Como se verifica en la tesis referida, el software de autoaprendizaje o de los sistemas de simulación se constituyen en herramientas que proporcionan niveles superiores de aprendizaje. Ello sin duda tiene -y tendrá- diversas fases. Para algunos inclusive la robótica pedagógica se constituye en una forma superior de la propia pedagogía informática facilitando una mayor complejidad de la propia automatización pedagógica.

\section{La informatización de la gestión administrativa}

La digitalización de la gestión y de los diversos procesos administrativos y académicos de las universidades ha sido una de las mayores transformaciones en la dinámica universitaria. Estos procesos de digitalización siguen una lógica no académica. La base es la gestión, buscando una organización más eficiente y una disminución de los costos de funcionamiento. Al tiempo la informatización está asociada a una dinámica más competitiva en términos de mercado, de la presión de los costos por las regulaciones públicas de calidad. También incide la complejización de la enseñanza que impone la introducción de sistemas de administración más automatizados. Los procesos de inscripción, de pago, de cobro, de registro de notas, de asignación de aulas, de contabilización de horas docentes, de historia laboral, de inventario, de procedimientos, etc., se expanden en la medida a medida que las instituciones aumentan de tamaño, de escala y que se diversifican en términos curriculares, académicos y locativos, impulsan una gestión más eficiente presionando por la introducción de sistemas informatizados en las universidades. Se informatiza para gestionar la complejidad organizacional, y para responder a las demandas competitivas del mercado así como a las presiones del aumento de los costos por la necesidad de aumentar la calidad.

Ello se ha dado con mayor intensidad en el sector privado en América Latina en la década pasada, asociado a la reducción de su tasa de crecimiento ante la expansión pública gratuita y la propia presión de los costos con el aumento de las exigencias de los sistemas de aseguramiento de la calidad. Al tiempo, la digitalización de la gestión administrativa y académica, contribuye a virtualizar y digitalizar los procesos de enseñanza. Ello se 
expresa por ejemplo en los niveles de complejidad de las páginas Web y de sus niveles de interactividad. Los diversos diagnósticos han ido mostrando el aumento de la interactibilidad en las plataformas de enseñanza virtuales. El avance desde la existencia de web sin interactividad, a la incorporación de la interacción informativa, a la interactividad consultiva y la interactividad comunicacional para llegar a niveles de interactividad transaccional, muestra un cambio que es al tiempo el soporte del desarrollo de niveles de enseñanza - aprendizaje virtuales con mejores niveles de calidad. El nivel de interacción de la web muestra al mismo tiempo el nivel de informatización de los procesos internos de las universidades en general y por ende de la incorporación de dinámicas y de pedagogías informáticas. En este sentido, hay una expresión en la web de los niveles de informatización de la gestión con la virtualización y la incorporación de pedagogías informáticas. El indicador de la webmetric muestra estas tendencias hacia una más amplia digitalización universitaria. También, en tanto las instituciones se van digitalizando y automatizando sus procesos administrativos, se viabiliza la propia virtualización del proceso de enseñanza, al tener recursos humanos capacitados, servidores, licencias, conectividad, etc.

\section{CONCLUSIÓN}

\section{La convergencia de los distintos procesos de virtualización}

En América Latina se está avanzando diferenciadamente en los procesos de digitalización y virtualización de la educación superior. En ella se constata globalmente la convergencia tecnológica y la lenta desaparición de la dualidad de modalidades, como resultado de los diversos caminos de la virtualización y digitalización. Se verifica un mayor avance a modelos de "blended learning" o educación semivirtual, que se gesta desde el paradigma a distancia o del paradigma presencial. Ambos sin duda tienen sus propias eficacias y limitaciones en términos educativos para contribuir a adquirir competencias. Esta convergencia digital está construyendo un nuevo paradigma educativo por competencias y digitales, como expresión de una nueva tecnología que está cambiando los procesos tradicionales de producción, distribución, intercambio y consumo en la mayor parte de los sectores de nuestras sociedades contemporáneas. La expansión de la virtualización de este modelo semivirtual tiene también restricciones. Por 
otro lado, la virtualización tiene limitaciones normativas en las autorizaciones gubernamentales y en el reconocimiento de las certificaciones internacionales. El otro modelo en la región tiene actualmente limitaciones a su expansión derivadas de la relativa ausencia de las aplicaciones informáticas, especialmente diseñadas para informatizar asignaturas y permitir un cambio a focalizarse en un enfoque curricular por competencias. En general, los software de simulación o de aplicaciones específicas tienen precios muy elevados que restringen su incorporación en los procesos de enseñanza en muchas universidades de la región. Hoy apenas algunas de las grandes universidades públicas de México y Brasil, o algunas instituciones privadas de élite logran adquirir muchas de estas herramientas informáticas. Hay sin duda una ventana de oportunidades a la producción de contenidos digitales. Tenemos muchos libros para transferir conocimientos en las distintas asignaturas, pero hay muy pocos software de base Web para crear competencias en las estructuras curriculares. En estos escenarios estará focalizada la convergencia digital y la educación virtual. Desde el campus virtual o desde el laboratorio informático, se desarrollan procesos de enseñanza y aprendizaje a través de aplicaciones informáticas que permitan un aprender haciendo, un nuevo rol del docente y un enfoque por competencias.

\section{REFERENCIAS}

Amador, Rocío. Educación y formación a distancia, México: Universidad de Guadalajara, 2001.

Bates A.W., T. Cómo gestionar el cambio educativo, España: Gedisa, 2001.

Berra, M. "Innovación tecnológica e innovación social. Nuevos modelos organizativos". Revista Gestión y Estrategia (1994). www.azc.uam.mx/publicaciones/gestion/Num6/art14.html

Castells, M. La sociedad en red: una visión global. Barcelona: Alianza, 2004.

Castells, M. La Galaxia Internet. Madrid: Plaza \& Janés, 2001.

Chacón, F. Reingeniería de la educación abierta y a distancia en América Latina, Venezuela: 1996. www.c5.cl/ieinvestiga/actas/ribie96/Reing/html

Villarroel, Armando (ed.). La Educación a Distancia: desarrollo y apertura. Caracas: ICDE - Universidad Nacional Abierta, 1996.

Daniel, J. Mega-universities \& knowledge media. Technology strategies for higher education. London: Kogan Page, 1998.

Instituto de la UNESCO Educación Superior para América Latina (IESALC). La educación superior virtual en América Latina y el Caribe. México: ANUIES, 2004. 
Instituto Internacional de la UNESCO para la Educación Superior en América Latina y el Caribe (IESALC) Informe sobre la Educación Superior en América Latina, Caracas: IESALC, 2006.

Facundo, Á. Educación Virtual en América Latina y el Caribe: Características y tendencias. Bogotá: Universidad de El Libertador, 2002.

García, Lorenzo, Marta Ruiz y Daniel Domínguez (comp.) De la educación a distancia a la educación virtual. España: Ariel, 2007.

Martínez, B. "Educación a distancia y educación virtual en América Latina", Congreso Virtual Educa, 2005.

Pastor Angulo, M. Educación, estandarización y tecnología. Contradicciones y tendencias. México: UDG Virtual, 2006.

Peters, Otto. La educación a distancia en transición. Nuevas tendencias y retos. México: Universidad de Guadalajara, 2002.

Rama, Claudio. Los postgrados de América Latina en la sociedad del conocimiento. México: Unión de Universidades de América Latina (UDUAL), 2007.

Rama, Claudio. Las tendencias de la educación superior en América Latina en el siglo XXI (desautonomización, despresencialización, desgratuitarización y desnacionalización). Lima: Asamblea Nacional de Rectores, 2008.

Rama, Claudio. La encrucijada de tendencias de la universidad latinoamericana. República Dominicana, UNICARIBE, 2010.

Rifkin, J. La era del acceso. La revolución de la nueva economía, Madrid: Paidós, 2000.

Silvio, J. Tendencias de la educación virtual en América Latina, Caracas: IESALC, 2003.

Toffler, A. y Toffler, H. La revolución de la riqueza, Barcelona: Debate, 2006.

UNESCO. Directrices en materia de calidad de la educación superior a través de las fronteras. París: UNESCO, 2006. 


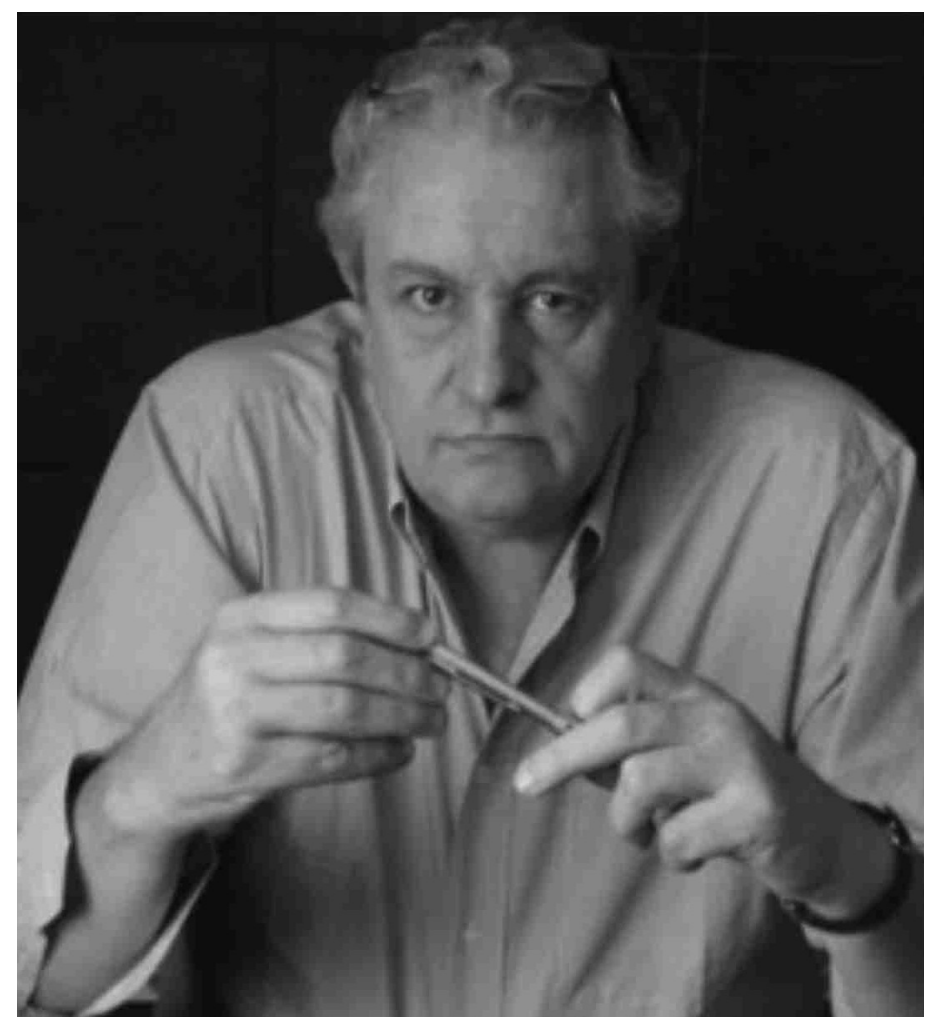

Fuente: http://www.ut.ac.id/icde2011/claudio-rama/

\begin{tabular}{l|l} 
& \\
\hline $\begin{array}{l}\text { Rama, Claudio. "Los caminos de las reformas. La virtualización universitaria } \\
\text { en América Latina”. Revista Historia de la Educación Latinoamericana. Vol. } 14 \\
\text { No. 19, (2012): }\end{array}$ & \\
\hline
\end{tabular}

